\title{
Anisotropic Transport of Electrons in $\mathrm{ZnS}$ Thin-Film Electroluminescence Displays
}

\author{
ZHENG XU \\ Institute of Optoelectronic Technology \\ Beijing Jiaotong University, Beijing 100044, China \\ and \\ Postdoctoral Working Station of Zhong-Huang San-Jin Ltd. \\ District of Economy and Technology Development, Tianjin, China
}

(Received November 18, 2003)

We study the anisotropic electronic transport property of ZnS-type thin-film electroluminescence displays by Monte Carlo simulation. The simulation contains an accurate and efficient description of the anisotropic band structure and various scattering mechanisms like phonon scattering and impurity scattering. The electronic transport processes in three devices with different ZnS-layer orientations are simulated. From the obtained energy population and average energy of electrons, we conclude that the $\langle 100\rangle$ direction is the best for electron acceleration under high electric field. We propose that new attempts in using this direction for ZnS-layer deposition will result in an improvement of the performance of thin-film electroluminescence displays.

PACS numbers: 78.60.Fi

\section{Introduction}

Thin-film electroluminescence displays (TFELDs) have been extensively developed during the past two decades since they offer a possible means of achieving a high-resolution, light-weight video display panel for computer terminals and television screens [1]. Yellow-emitting ZnS:Mn TFELDs have been commercially available for more than ten years. However, the development of TFELD into a full-color display has been postponed due to the poor brightness and efficiency of blue emission. In order to overcome this problem, deeper understanding of material, device structure and electroluminescence mechanism is highly desired.

Beside the promising application possibilities, the electroluminescence process is also an ideal laboratory for studying a number of important physics processes in solids like high-field transport, impact excitation, impact ionization, and 
recombination. In a TFELD, a thin phosphor layer, e.g. ZnS, is sandwiched between two insulating layers. Outside each insulating layer, an electrode layer is deposited. When a voltage is applied across the two electrodes, the electrons trapped in the interface states between the phosphor layer and the insulating layer are emitted into the phosphor layer due to the field-induced tunneling process. These electrons are thus accelerated in the high electric field in the phosphor layer. Electrons with enough energy impact the luminescence centers that are intentionally doped into the phosphor layer. Some of the centers may be excited by this impact process, leading to the emission of light for the display applications. Among these processes, the acceleration of electron in the phosphor layer is a key process since it determines whether the electrons can obtain enough energy for impact excitation.

In previous studies, the mostly used material for the phosphor layer is ZnS. The growth direction of ZnS layer in these devices is the $\langle 111\rangle$ direction. Accordingly, the electric field is applied along the $\langle 111\rangle$ direction. Thus, the electrons are accelerated along this direction. No attempt has been made in growing the ZnS layer along other directions for comparison of the performances. However, since the transport process of electrons is direction-dependent, devices with a $\mathrm{ZnS}$ layer grown along other directions may show different transport performances. To examine this possibility of optimizing the TFELD performance, we study for the first time the directional property of electron transport in ZnS-based TFELD by Monte Carlo simulation. We find that when the ZnS layer is grown along $\langle 100\rangle$ direction, the energy which the electrons obtained from the acceleration process is significantly larger than that grown along other directions.

\section{Monte Carlo simulation}

The Monte Carlo (MC) simulation has been widely used in studying the carrier transport in solid states [2-7]. It simulates the motion of individual carriers as they move in the material under the influence of external fields and are scattered by a number of mechanisms. In a classical MC simulation, the scattering event is assumed to be instantaneous. Thus, the motion is composed by a series of free-flight processes terminated by the scattering events. The free flight is a scattering-free process, so the motion is described as

$$
\frac{\mathrm{d}}{\mathrm{d} t}(\hbar \boldsymbol{k})=-e \boldsymbol{E},
$$

where $\boldsymbol{k}$ and $\boldsymbol{E}$ are the wave vector of electron and the strength of the external electric field, respectively. The time scale that the electron can fly ballistically depends on how frequently do the scattering events happen. Therefore in the simulation the duration of each free-flight process is chosen randomly according to the total scattering rate, $\Gamma$, as

$$
t_{\mathrm{f}}=-\frac{1}{\Gamma} \ln (r),
$$

where $r$ is a random number evenly distributed in $[0,1]$. 
At the end of each free flight, the position and velocity of each electron is calculated. One type of scattering mechanism is chosen to happen from all the scattering mechanisms considered in the simulation. The choice is made randomly, according to the relative scattering rates of these mechanisms. The final state of electron after this scattering event is easily obtained from the electron state at the end of the free-flight process and the mechanism of the scattering. This state is then used as the initial state of the next free-flight process, which is terminated by the next scattering event. By repeating this procedure, the motion of electrons in the ZnS layer is simulated.

In order to correctly describe the motion of electron in $\mathrm{ZnS}$ conduction band, the band structure of $\mathrm{ZnS}$ must be modeled with high accuracy. In previous simulations, both parabolic [8] and nonparabolic [9] curves have been adopted to approximate the band structure. A more rigid description is the full-band model based on calculations by empirical pseudopotential method [10,11]. In the present simulation, we exploit an analytical approach to describe the band structure. We take the data of band structure obtained by empirical pseudopotential calculation, and fit it by polynomials. The Brillouin zone is divided into several extended valleys. In each valley, the dispersion relation is fitted by a two-piece polynomial. Figure 1 shows the band structure calculated by empirical pseudopotential method (solid line) and the fitted curves by the polynomials (points). It is clear that the band structure is accurately described in this model. The advantage of this approach is that the band structure is accurate and still analytical. This makes the simulation more efficient [12]. From Fig. 1, it is also clear that the band structure is quite different along different directions from $\Gamma$ point. This implies different effective mass, density of states, and scattering rates when the electrons are accelerated along different directions. This is the origin of the anisotropic transport property of electrons in ZnS TFELD examined here.

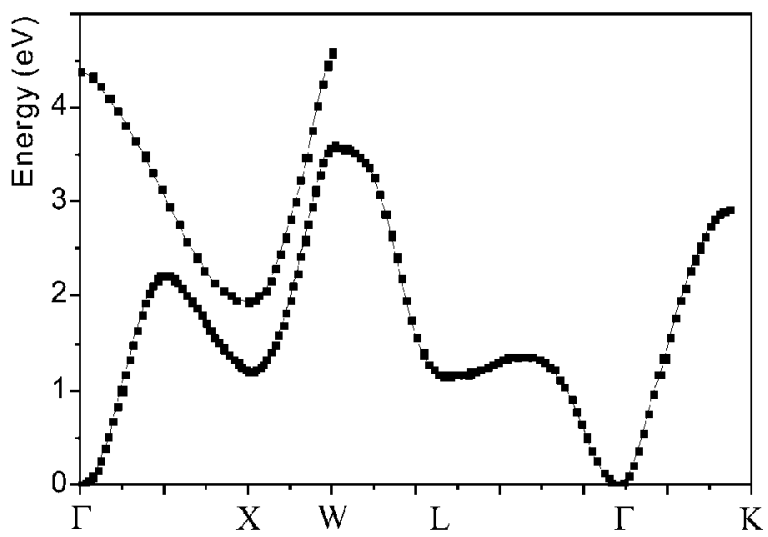

Fig. 1. Band structure of ZnS calculated by empirical pseudopotential method (solid line) and the corresponding fits (points) by two-piece polynomials. 
The electron-phonon scattering mechanisms considered in the simulation include acoustic-phonon emission, acoustic-phonon absorption, optical-phonon emission, optical-phonon absorption, and intervalley scattering. The rates of these scattering mechanisms are calculated by the Fermi golden rule [13]. Considering the typical operating conditions of TFELD, the phonon scattering rates are calculated at a sample temperature of $300 \mathrm{~K}$. Beside these phonon scattering mechanisms, we also include the scattering of electron by the charged centers like impurities and lattice vacancies, which generally exist in TFELD [14].

\section{Simulation results}

Using the method described above, we simulate the electron transport process in ZnS-type TFELDs with different growth direction of $\mathrm{ZnS}$ layer. Ten-million electrons are simulated simultaneously in order to get the statistic result with enough high accuracy. In the simulation, the initial kinetic energy of electrons is set to be thermal energy, $k_{\mathrm{B}} T$. This is relevant since the electrons are injected into the ZnS layer through the interface state with small energy. The cold electron is accelerated by the electric field during a transient process [15]. After this process, the electron obtains its steady-state energy with which the energy gain from the electric field is balanced by the loss to phonon system. Here, we examine the steady state of electron, rather than the transient process. This is relevant since the transient process only happens within a region of about $50 \mathrm{~nm}$, a small part of the $\mathrm{ZnS}$ layer [16]. Thus, the device performance is mainly determined by the steady energy of electrons.

Figure 2 shows the simulated steady energy distribution of electrons in TFELDs with a ZnS layer grown along the direction of $\langle 100\rangle$ (solid line), $\langle 110\rangle$ (dashed line), and $\langle 111\rangle$ (dotted line). From these curves, it is clear that the

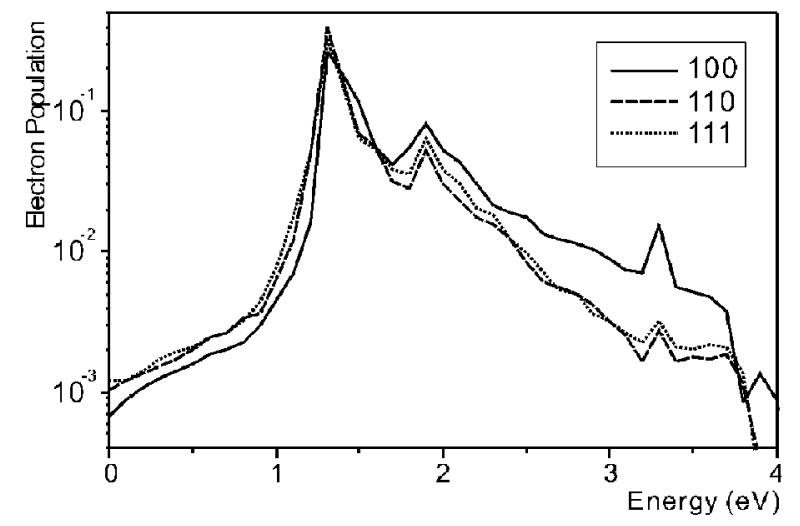

Fig. 2. Steady energy distribution of electrons obtained by the simulation for TFELDs with a ZnS layer grown along the direction of $\langle 100\rangle$ (solid line), $\langle 110\rangle$ (dashed line), and $\langle 111\rangle$ (dotted line). The electric field strength is $3 \mathrm{MV} / \mathrm{cm}$. 
electrons populate more high-energy states in the device with a growth direction of $\langle 100\rangle$ than that along other directions. Small peaks around 1.8 and $3.3 \mathrm{eV}$ in the population curves are induced by the accumulation of electrons before intervalley-scattering processes [17].

Beside the energy distribution, the electron distribution on different energy valleys in the conduction band is also deduced from the simulation. With these distribution functions, we obtain the average values of the kinetic energy and total (kinetic and potential) energy of electrons under different conditions. Figure 3

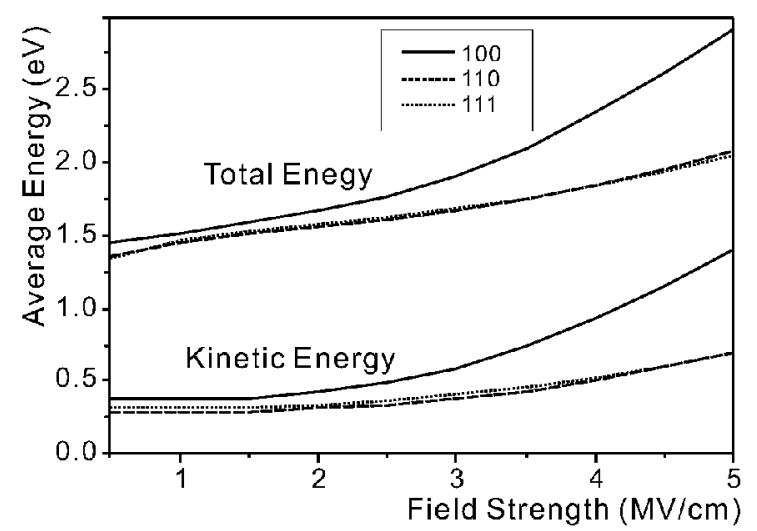

Fig. 3. Average energy as function of the electric field strength with the field along different directions. Both the kinetic energy and the total energy are shown.

shows the average kinetic energy and total energy of electron as function of field strength for three different devices with different growth direction of the $\mathrm{ZnS}$ layer. In consistency with the energy distribution functions shown in Fig. 2, the energy of electron is the highest in the device with $\langle 100\rangle$ direction of $\mathrm{ZnS}$ layer. This shows that the $\langle 100\rangle$ direction has the best acceleration performance. At low field strength, the difference of the energy is not much pronounced among the three types of the devices. With increasing the field strength, the difference becomes more and more pronounced. Generally, the ZnS layer is grown along $\langle 111\rangle$ direction in the TFELDs. Our simulation shows that this direction is not the best choice for electron acceleration performance. Due to the key role the acceleration process played in the whole electroluminescence process and the importance of the electron energy on the luminescence efficiency, we anticipate an improvement of the device performance by changing the growth direction of the $\mathrm{ZnS}$ layer.

\section{Conclusion}

We study the anisotropic transport property of ZnS-type TFELDs by MC simulation. The detailed and accurate description of $\mathrm{ZnS}$ conduction-band by polynomials is introduced into the simulation with high efficiency. This band model 
fully describes the anisotropic property of the band structure, which is the source of the anisotropic transport of electrons. Both the phonon scattering and the impurity scattering mechanisms are included in the simulation. From the simulation, we obtained the steady energy distribution of electrons, and found that in the device with a $\langle 100\rangle$ oriented $\mathrm{ZnS}$ layer, more electrons populate high-energy states. Quantitatively, we obtain the average energy of electrons under different electric field strength for the devices with different $\mathrm{ZnS}$ orientations. The acceleration performance along $\langle 100\rangle$ direction is significantly better than that along other directions, especially under high electric field. Since the electron energy is the key parameter in determining the device efficiency and brightness, we anticipate that attempts in growing the $\mathrm{ZnS}$ along $\langle 100\rangle$ direction, rather than the currently used $\langle 111\rangle$ direction, may lead to a new improvement in the device performance.

\section{Acknowledgment}

The author acknowledges financial supports by NSFC (10374001), China Postdoctoral Science Foundation (2003034324), 973(2003CB314707), RFDP(20020004004), NSFB(2032015) and Paper Foundation of BJTU.

\section{References}

[1] Y.A. Ono, Electroluminescence Display, World Scientific, Singapore 1995.

[2] C. Jacoboni, P. Lugli, The Monte Carlo Method for Semiconductor Device Simulation, Springer, USA 1989.

[3] H. Zhao, B. DalDon, S. Moehl, H. Kalt, K. Ohkawa, D. Hommel, Phys. Rev. B 67, 035306 (2003).

[4] E. Bringuier, J. Appl. Phys. 66, 1314 (1989).

[5] K. Brennan, J. Appl. Phys. 64, 4024 (1988).

[6] H. Zhao, S. Moehl, H. Kalt, Phys. Rev. Lett. 89, 097401 (2002).

[7] H. Zhao, Z. Xu, Y.S. Wang, Y.B. Hou, X.R. Xu, Displays 21, 143 (2000).

[8] R. Mach, G.O. Muller, J. Cryst. Growth 101, 967 (1990).

[9] K. Bhattacharyya, S.M. Goodnick, J.F. Wager, J. Appl. Phys. 73, 3390 (1993).

[10] M. Dur, S.M. Goodnick, S.S. Pennathur, J.F. Wager, M. Reigrotzki, R. Pedmer, J. Appl. Phys. 83, 3176 (1998).

[11] R. Bellottin, K. Brennan, R. Wang, P.P. Ruden, J. Appl. Phys. 83, 4765 (1998).

[12] H. Zhao, Y.S. Wang, X.R. Xu, Acta Phys. Pol. A 98, 123 (2000).

[13] H. Zhao, Y.S. Wang, Z. Xu, X.R. Xu, Czechoslovak J. Phys. 50, 1159 (2000).

[14] H. Zhao, Y.S. Wang, Z. Xu, X.R. Xu, Semicond. Sci. Technol. 14, 1098 (1999).

[15] H. Zhao, Y. Wang, Z. Xu, X. Xu, J. Phys., Condens. Matter 11, 2145 (1999).

[16] H. Zhao, Y.B. Hou, Y.S. Wang, X.R. Xu, Phys. Scr. 65, 500 (2001).

[17] H. Zhao, Y.S. Wang, Z. Xu, X.R. Xu, Acta Phys. Pol. A 96, 475 (1999). 